\title{
Erinnerte gentes. Geschichtsgedächtnis für das spätere Mittelalter
}

Die Geschichte frühmittelalterlicher Ethnogenesen wurde in immer neuen wissenschaftlichen Anläufen erforscht und geschrieben. Die Beiträge dieses Bandes spiegeln die wichtigsten Perspektiven und zeigen die neuen Einsichten, die von den Forschergruppen um Jörg Jarnut in den letzten Jahren gewonnen wurden. In diesem Aufsatz soll nicht etwa versucht werden, aus Quellen des 14. und 15. Jahrhunderts auch nur Splitter von Originalität in die hochkomplexe Forschung über Völker, Reiche und Namen zwischen Spätantike und Frühmittelalter einbringen zu wollen.

Hier geht es um die Prägekraft spätmittelalterlicher Verformungen für unsere Suchstrategien nach den Wurzeln des frühmittelalterlichen Europa. Die Lektüre vieler absonderlich anmutender Quellen eröffnet die iterative Wucht von Stereotypen, die keineswegs erst der modernen Nationalgeschichte entsprangen. ${ }^{1}$ Nicht alle wichtigen Deutungswege entstanden erst im 19. und 20. Jahrhundert aus völkischen Verformungen der Vergangenheit, wie dies lange geglaubt wurde. ${ }^{2}$ Entscheidende Weichenstellungen für die fortwährende Nationalisierung des früheren Mittelalters lieferte bereits die Geschichtsbenutzung des ausgehenden Mittelalters. ${ }^{3}$ Zwar ist die Tragfähigkeit glatter Traditionslinien vom Humanismus zum Nationalsozialismus vielfach hinterfragt worden, so dass auf bloße Wiederholung solcher Behauptungen und Differenzierungen verzichtet werden kann. Die Modi des 14. bis 16. Jahrhunderts im Umgang mit früheren Völkern, Reichen und Namen lassen indes erstaunliche Prägekräfte hervortreten. Sie tauchen aktuelle Debatten, wie sie mit dem latenten Faschismusvorwurf gegenüber der deutschsprachigen ethnogenetischen Forschung von amerikanischen Wissenschaftlern geführt werden, in neues Licht. Aus der Fülle der Überlieferung, die es hier nicht angemessen auszubreiten,

1 Methodisch wegweisend Graus, František: Lebendige Vergangenheit. Überlieferungen im Mittelalter und in den Vorstellungen vom Mittelalter, Köln/Wien 1975. Vgl. auch FlaCKe, Monika (Hg.): Mythen der Nationen. Ein europäisches Panorama, Berlin 1998.

2 Beck, Heinrich/Geuenich, Dieter/Steuer, Heiko/Hakelberg, Dieter (Hg.): Zur Geschichte der Gleichung ,germanisch - deutsch'. Sprache und Namen, Geschichte und Institutionen (RGA, Ergbd. 34), Berlin/New York 2004. Vgl. auch OвеRкrome, Willi: Volksgeschichte. Methodische Innovation und völkische Ideologisierung in der deutschen Geschichtswissenschaft 1918-1945 (Kritische Studien zur Geschichtswissenschaft 101), Göttingen 1993; GROTHE, Ewald: Zwischen Geschichte und Recht. Deutsche Verfassungsgeschichtsschreibung 1900-1970 (Ordnungssysteme. Studien zur Ideengeschichte der Neuzeit 16), München 2005; NAGEL, Anne Christine: Im Schatten des Dritten Reichs. Mittelalterforschung in der Bundesrepublik Deutschland 1945-1970 (Formen der Erinnerung 24), Göttingen 2005.

3 BorChardT, Frank L.: German Antiquity in Renaissance Myth, Baltimore/London 1971; HirsCHI, Caspar: Wettkampf der Nationen. Konstruktionen einer deutschen Ehrgemeinschaft an der Wende vom Mittelalter zur Neuzeit, Göttingen 2005. 
sondern schlaglichtartig zu präsentieren gilt, wähle ich drei Weisen des Geschichtsgedächtnisses aus. Vordergründig pointillistisch anmutend, deuten sie gleichwohl die Breite spätmittelalterlicher Begehrlichkeiten auf die richtige und gute Erinnerung an.

\section{Sächsische Aneignungen}

Die lateinischen Verschriftlichungen der sächsischen Stammes-Origo ${ }^{4}$ waren im ausgehenden Mittelalter weitgehend präsent. ${ }^{5}$ Freilich wurden die Geschichten von Ethnogenese, Landnahme und Thüringerkrieg im Lauf des Mittelalters um kühne Rückgriffe auf die griechische und römische Antike angereichert.

Mit dem Aufstieg der Liudolfinger zum Königtum und mit dem damit einhergehenden Rang des sächsischen Volkes im ostfränkischen Reich wuchs die Notwendigkeit neuer Legitimationsstrategien. ${ }^{6}$ Die Verknüpfung des römischen Kaisertums mit dem ostfränkischen Königtum durch die drei ottonischen Kaiser kreierte den Nachgeborenen Erinnerungsräume, die zum mutigen Griff auf die globale Kaisergeschichte ermutigten. So fundierte man im Spätmittelalter die eigene Existenz nicht mehr nur aus der sächsischen Landnahme oder der ottonischen Herrschaft, sondern entwarf im humanistischen Wettstreit neue Gründungsmythen, nach denen Julius Caesar selbst die großen sächsischen Städte gegründet habe. Solch späte Bewusstseinsschichten schienen der aufgeklärten Moderne so abstrus, dass sie kaum das Interesse der Forschung finden konnten. Diese konzentrierte sich vielmehr in typisch historistischer Manier auf die frühesten schriftlichen Zeugnisse zur sächsischen Frühgeschichte aus dem 9. und 10. Jahrhundert. Die Sachsengeschichte Widukinds von Corvey lieferte einer kritischen Mediaevistik in weit höherem Maß Zugänge zur frühmittelalterlichen Wirklichkeit als die spätmittelalterlichen Fabeln vom römischen Ursprung. ${ }^{7}$ Erst in neuerer Zeit mehrten sich die Zweifel am Umgang mit den frühen Erinnerungstexten, die nun selbst wiederum als Zeugnisse permanenter Verformung über die Jahrhunderte gelesen wer-

4 Vgl. die Hinweise bei Springer, Matthias: Die Sachsen (Urban-Taschenbücher 598), Stuttgart 2004.

5 Vgl. Nass, Klaus: Die Reichschronik des Annalista Saxo und die sächsische Geschichtsschreibung im 12. Jahrhundert (MGH Schriften 41), Hannover 1996; Weddige, Hilkert: Heldensage und Stammessage. Iring und der Untergang des Thüringerreiches in Historiographie und heroischer Dichtung (Hermaea N. F. 61), Tübingen 1989; Herkommer, Hubert (Hg.): Das Buch der Welt. Kommentar und Edition zur ,Sächsischen Weltchronik' Ms. Membr. I 90 Forschungs- und Landesbibliothek Gotha, Luzern 2003; Andermann, Ulrich: Albert Krantz. Wissenschaft und Historiographie um 1500 (Forschungen zur mittelalterlichen Geschichte 38), Weimar 1999.

6 Zusammenfassend Puhle, Matthias (Hg.): Otto der Große, Magdeburg und Europa, 2 Bde., Mainz 2001; Schneidmüller, Bernd/Weinfurter, Stefan (Hg.): Ottonische Neuanfänge. Symposion zur Ausstellung, Otto der Große, Magdeburg und Europa', Mainz 2001.

7 Vgl. die Darstellung in Patze, Hans (Hg.): Geschichte Niedersachsens, Bd. 1: Grundlagen und frühes Mittelalter (Veröffentlichungen der Historischen Kommission für Niedersachsen und Bremen 36), Hildesheim ${ }^{2} 1985$. 
den. ${ }^{8}$ Gewiss - Widukind stand der sächsischen Ethnogenese zeitlich wesentlich näher als die Magdeburger Schöppenchronik. Hier soll auch keine Lanze für eine quellenkritische Beliebigkeit gebrochen werden, in der jede historische Quelle als bloße literarische Fiktion entwertet wird. Gleichwohl muss man sich die zeitlichen Abstände als Korrektur überkommener Geschichtsmodelle vor Augen halten.

Der sächsische Mönch, der im letzten Lebensjahrzehnt Kaiser Ottos des Großen († 973) schrieb, war tatsächlich gar nicht so viel näher an der sächsischen Landnahme der Frühzeit. Er erzählte aus seinen historischen Interessen an der Glorifizierung seines Volkes angesichts imperialer Herausforderungen ${ }^{9}$ andere Geschichten als seine Nachfolger. Diesen war im ausgehenden Mittelalter der Rückgriff auf die antiken Traditionen des Heiligen Römischen Reichs inzwischen so selbstverständlich, dass im konkurrierenden Kommunikationskontext interurbanen Selbstbewusstseins auch für norddeutsche Städte der Rückgriff auf Caesar als Gründergestalt notwendig wurde. Zur genaueren Auslotung der Ziele und der Entwicklungen wenden wir uns im Gefolge der Untersuchung von Brigitte Funke der Magdeburger Schöppenchronik und der Cronecken der Sassen zu. ${ }^{10}$

In einer ersten Textstufe formulierte der Magdeburger Stadtschreiber Heinrich von Lammesspringe die Schöppenchronik vor 1372; die erste erhaltene Handschrift entstand kurz nach $1468 .{ }^{11}$ Die anonyme Cronecken der Sassen ist uns in einem Mainzer Frühdruck von Peter Schöffer aus dem Jahr 1492 erhalten; ${ }^{12}$ die Chronik wurde zur Braunschweiger Historiographie des ausgehenden Mittelalters gerückt. ${ }^{13}$

Die Komposition der Schöppenchronik zielte auf das Magdeburger Schöffenkolleg und erfasste die Geschichte aus Magdeburger Perspektiven in drei großen

8 FRIED, Johannes: Der Schleier der Erinnerung. Grundzüge einer historischen Memorik, München 2004.

$9 \mathrm{Zu}$ den literarischen Zielen immer noch grundlegend Beumann, Helmut: Widukind von Korvei. Untersuchungen zur Geschichtsschreibung und Ideengeschichte des 10. Jahrhunderts (Abh. zur Corveyer Geschichtsschreibung 3 = Veröffentlichungen der Historischen Kommission des Provinzialinstituts für westfälische Landes- und Volkskunde X 3), Weimar 1950.

10 Funke, Brigitte: Cronecken der sassen. Entwurf und Erfolg einer sächsischen Geschichtskonzeption am Übergang vom Mittelalter zur Neuzeit (Braunschweiger Werkstücke 104), Braunschweig 2001.

11 Edition von Janicke, Karl: Die Chroniken der deutschen Städte, Bd. 7: Magdeburg 1, Leipzig 1869.

12 Chronecken der Sassen: Gesamtkatalog der Wiegendrucke, 7 Bde., Leipzig 1925-1938, Nr. 4963; Borchling, Conrad/Claussen, Bruno (Bearb.): Niederdeutsche Bibliographie. Gesamtverzeichnis der niedersächsischen Drucke bis zum Jahre 1800, 3 Bde., Neumünster 1931-1957, Nr. 197. Ein Verzeichnis nachweisbarer Drucke mit 93 Nummern bei Funke (Anm. 10), S. 302-304, vgl. dort auch Anm. 1076 zu weiteren Exemplaren.

13 Dazu Ehlers, Joachim: Historiographie, Geschichtsbild und Stadtverfassung im spätmittelalterlichen Braunschweig, in: EHLERs, Joachim: Ausgewählte Aufsätze (Berliner Historische Studien 21), Berlin 1996, S. 521-562. Aus der reichen Forschung zum Braunschweiger Stadtschreiber Hermen Bote vgl. Blume, Herbert/Rohse, Eberhard (Hg.): Hermann Bote. Städtisch-hansischer Autor in Braunschweig 1488-1988. Beiträge zum Braunschweiger Bote-Kolloquium 1988 (Frühe Neuzeit 4), Tübingen 1991. Zum geistigen Leben in Braunschweig im Übergang vom Mittelalter zur Neuzeit Haucap-Nass, Anette: Der Braunschweiger Stadtschreiber Gerwin von Hameln und seine Bibliothek (Wolfenbütteler Mittelalter-Studien 8), Wiesbaden 1995; Kirschner, Carola: Hermen Bote. Städtische Literatur um 1500 zwischen Tradition und Innovation, Essen 1996. 
Teilen, der sächsischen Frühzeit bis zu Otto dem Großen, der Epoche von Otto dem Großen bis zur Großen Pest von 1350 und der unmittelbaren Zeitgeschichte des Verfassers im dritten Viertel des 14. Jahrhunderts. Während die bisher wenig beachtete Sächsische Weichbildchronik die sächsische bzw. die Magdeburger Rechtsentwicklung aus dem Kaiserrecht von Konstantin, Karl dem Großen, Otto dem Großen und Otto II. herleitete und damit den eigenen Lebensort in imperiale Traditionen fügte, ${ }^{14}$ nahm die Schöppenchronik dezidiert die Magdeburger Gründungslegende durch Caesar, die sächsische Stammessage und die Geschichte von der Begründung des Kurfürstenkollegs durch Karl den Großen auf. Damit verwob sich die Darstellung der älteren Geschichte mit aktuellen Magdeburger Erfahrungen aus Kontakten mit dem Hof Kaiser Karls IV. im Jahr 1359 und mit der Fixierung des Kurkollegs durch die Goldene Bulle von 1356. ${ }^{15}$

Konstitutiv für das lange Geschichtsbewusstsein wurde die zeitliche Überwindung sächsischer Geschichte durch ältere kommunale Traditionen der Elbmetropole. Am Anfang der Magdeburger Geschichte standen nicht die Sachsen, sondern Julius Caesar aus dem Geschlecht des Aeneas. Bis zur Elbe sei er gekommen, um hier das Volk der Thüringer zu bezwingen und einen Tempel für die Göttin Diana zu erbauen. In seiner Sprache hieß sie Parthenya, so dass der Platz den Namen Partenopolis erhielt. Weil dort viele Mägde der Göttin dienten, kam der Name Megedeborch auf. ${ }^{16}$ Erst vierhundert Jahre später seien die Sachsen dorthin gekommen und hätten die Thüringer vertrieben. ${ }^{17}$ Magdeburgs Beginn verband sich also mit dem Anfang des römischen Kaisertums, später mit dem ostfränkischen Königtum und schließlich mit dem ottonischen Kaisertum. Mit einer solchen Stadtgeschichte, die das imperiale Handeln Ottos des Großen und Ottos II. mit der Gründung durch Julius Caesar verknüpfte, ${ }^{18}$ konnte Magdeburg mit dem historischen Selbstbewusstsein oberdeutscher Städte wie Augsburg, Regensburg oder Nürnberg angemessen mithalten. ${ }^{19}$

14 Daniels, A. von/Gruben, Fr. von (Hg.): Das Sächsische Weichbildrecht. Jus municipale saxonicum, Bd. 1 (Rechtsdenkmäler des deutschen Mittelalters 1), Berlin 1858, S. 32 f., S. 37.

15 Dazu Funke (Anm. 10), S. 74.

16 Do Julius desse land bedwungen hadde, he buwede vele borgen und vesten in dit land, uppe dat he dat volk dwingen mochte. he buwede Yleborch Kueffhusen Luneborch und ok ander vesten vele. he buwede hir, dar dusse stad steit, ein borch und einen tempel in de ere siner godinne Dianen, de heit he na siner tungen Parthenya. dar af gaf he dusser stad den namen Partenopolis. he gaf in den tempel der godinne vele megede to denste, dar af wart sedder de name Megedeborch. dat sulve dudet ok Partenopolis, Die Magdeburger Schöppenchronik (Anm. 11), S. 7.

17 Do Julius in dat land quam, do erhof he sik siner kraft und toch hir in disse land wente an de Elve, und bedwang dat volk dat in dusseme lande sat. wat volkes dat was und wo se heiten, wil ik di seggen. id weren Doringe, wente hir na over veirhundert jare edder bi der mate kemen de Sassen to lande und dreven de Doringe ut dissen lande, als hir na geschreven steit, Die Magdeburger Schöppenchronik (Anm.11), S. 7.

18 Schneidmüller, Bernd: Magdeburg und das geträumte Reich des Mittelalters, in: SCHneidMÜLleR, Bernd/Weinfurter, Stefan (Hg.): Heilig - Römisch - Deutsch. Das Reich im mittelalterlichen Europa, Dresden 2006, S. 10-43.

19 Schмidt, Heinrich: Die deutschen Städtechroniken als Spiegel des bürgerlichen Selbstverständnisses im Spätmittelalter (Schriften der Historischen Kommission bei der Bayerischen Akademie der Wissenschaften 3), Göttingen 1958; Weber, Dieter: Geschichtsschreibung in Augsburg. Hektor Mülich 
Der Chronist erzählt, Heinrich I. habe die Städte und Bürger durch die Verleihung des Heerschildrechts in die feudale Welt des Mittelalters eingebunden. ${ }^{20}$ Die Ordnung des Reichs mit Kurfürstenkolleg und Königswahl führte die Schöppenchronik auf Karl den Großen zurück. Er habe die Königswahl durch die Kurfürsten eingerichtet und als erste Kurfürsten die Erzbischöfe von Köln, Trier und Mainz sowie den Pfalzgrafen bei Rhein bestimmt. Als die Franken für ihre Italienpolitik sächsische Hilfe benötigten, hätten sie diese durch die Erweiterung des Kurfürstenkollegiums um den Herzog von Sachsen und den Markgrafen von Brandenburg erlangt. ${ }^{21}$ Hinzu trat die beliebte Geschichte vom Burgenbau Heinrichs I. gegen die Ungarn, in spätmittelalterlicher Übersetzung als Städtebau verstanden. Wie andere norddeutsche Quellen lehrte die Magdeburger Schöppenchronik, dass alle öffentlichen Versammlungen und die ritterlichen Turniere auf Befehl Heinrichs I. in Städten abzuhalten seien. ${ }^{22}$ Bis in die frühe Neuzeit bildete sich eine feste Folge von 36 glanzvollen Ritterfesten des Mittelalters. Der christlichen Ritterschaft angeblich von Heinrich I. nach seinem Sieg über die Ungarn gestiftet, soll das erste große Turnier nach dieser Tradition 938 in Magdeburg stattgefunden haben. ${ }^{23}$ Die Sachsen traten also später in die weitaus ältere Stadtgeschichte mit ihren imperialen Anfängen ein; aus der Stadtgeschichte entstanden Volksgeschichte, Reichsgeschichte, Weltgeschichte.

Auf die Magdeburger Schöppenchronik und auf die Sächsische Weltchronik in der Tradition der Frutolf-Ekkehard-Überlieferung griff im ausgehenden 15. Jahrhundert die Cronecken der Sassen zurück. ${ }^{24}$ Hier stand das Sachsenvolk im Fokus, das über alle dynastische oder territoriale Zerstückelung des Landes die einheitliche Grundlage historischer Identität bot. Während die Schöppenchronik im 14. Jahrhundert noch Caesars Städtegründungen von der späteren sächsischen Geschichte trennte, schuf die Cronecken glatte Kontinuitäten und stellte die römische Vorzeit deutlicher in die eigene Vorgeschichte hinein. Caesar gründete angeblich sieben

und die reichsstädtische Chronistik des Spätmittelalters, Augsburg 1984; SCHNEIDER, Joachim: Anfänge in der Stadtgeschichte. Über Legenden in der mittelalterlichen Nürnberger Stadtchronistik und ihren historischen Auskunftswert, in: Mitteilungen des Vereins für Geschichte der Stadt Nürnberg 87 (2000), S. 5-46; Meyer, Carla: Die Stadt als Thema. Nürnbergs Entdeckung in Texten um 1500 (Mittelalter-Forschungen 26), Ostfildern 2009.

20 Vgl. SChneidmüller, Bernd: „Dem Heiligen Römischen Reich zu Ehren“. Sächsische Städte und das Reich im späten Mittelalter, in: Puhle, Matthias (Hg.): Hanse - Städte - Bünde. Die sächsischen Städte zwischen Elbe und Weser um 1500, Bd. 1 (Magdeburger Museumsschriften 4), Magdeburg 1996, S. 45-61.

21 Magdeburger Schöppenchronik (Anm. 11), S. $44 f$.

22 Ibid., S. 43. So auch die Chronecken der Sassen (Anm. 10), fol. 54v. Vgl. Stackmann, Karl: Die Stadt in der norddeutschen Welt- und Landeschronistik des 13. bis 16. Jahrhunderts, in: FLECKENSTEIN, Josef/Stackmann, Karl (Hg.): Über Bürger, Stadt und städtische Literatur im Spätmittelalter. Bericht über Kolloquien der Kommission zur Erforschung der Kultur des Spätmittelalters 1975-1977 (Abh. der Akademie der Wissenschaften in Göttingen. Philologisch-historische Klasse, III 121), Göttingen 1980, S. 289-310, hier S. 301; Funke (Anm. 10), S. 154 f.

23 Das große Buch der Turniere. Alle 36 glanzvollen Ritterfeste des Mittelalters. Die Bilderhandschrift des Codex Rossianus 711 im Besitz der Biblioteca Apostolica Vaticana. Einführung und Kommentar von Lotte Kurras, Stuttgart/Zürich 1996, fol. 9v-12r.

24 Die Belege bei Funke (Anm. 10), S. 105. 
Burgen zu Ehren der sieben römischen Taggötter. So verwoben sich Wolgast mit Merkur, die Eresburg (Marßborch) mit Mars, Hamburg (Henborch) mit Jupiter, Lüneburg mit Luna, die Harzburg mit Saturn, Salzwedel mit Sol und Magdeburg mit Venus. Karl der Große, der Apostel wie der Zuchtmeister der Sachsen, zerstörte diese heidnischen Kultstätten und gründete an ihrer Stelle christliche Kirchen. Nach der Cronecken soll Karl bei Tassilos Unterwerfung sogar die Bayern christianisiert haben. ${ }^{25} \mathrm{Da}$ der große Karolinger mit Widukind auch die sächsische Herzogsreihe einrichtete und die Edelinge als Stammväter späterer Fürstenhäuser - wie der Herzöge von Sachsen oder Braunschweig, der Markgrafen von Brandenburg oder der Fürsten von Anhalt - zu Grafen und Herren einsetzte, wurde er zum eigentlichen Schöpfer aller sächsischen Ordnung. ${ }^{26}$

So stilisierte die Cronecken Karl den Großen zur eigentlichen Integrationsfigur der Sachsen zwischen römisch-heidnischer Antike und Christianisierung. Heute könnte die historische Forschung einer solchen Kernthese wie ,Am Anfang war Karl der Große‘ wieder bereitwilliger folgen als ältere landesgeschichtliche Deutungsansätze, die noch die frühmittelalterliche Ethnogenese mit dem Aufstieg des sächsischen Volkes im 10. Jahrhundert gegen jede karolingische Überformung zusammenfügten. ${ }^{27}$ Die Ausgrenzung des fränkischen Zugriffs auf die sächsische Geschichte, die Widukind von Corvey vornahm, hatte in der Folge keinen Bestand mehr. Dem europäischen Zauber der Herrschergestalt Karls des Großen ${ }^{28}$ verfielen auch die Sachsen, denen die Zwangschristianisierung ihrer Vorfahren weniger wichtig wurde als deren Einbindung in den Kosmos des christlichen Europa. Aus den Deutungssehnsüchten des 14. und 15. Jahrhunderts ließen die Chroniken also in je eigener Weise das sächsische Volk in der Kaiser- oder Weltgeschichte aufgehen.

Deutlich wurde das schon in der Sächsischen Weltchronik, im 13. Jahrhundert vielleicht in Magdeburg entstanden. Sie wählte am Ende die neun wichtigsten Ereignisse der Weltgeschichte von Christi Geburt bis 1229 aus: Zerstörung des Tempels in Jerusalem - Herrschaft Konstantins des Großen - Herrschaft Karls des Großen - Herrschaft Ottos des Großen und Errichtung Magdeburgs (Van goddes bord over DCCCC und XXXVII iar ward Otto de grote keiser XXXVIII iar. In sineme anderen iare buwede he Maideburg) - Eroberung Jerusalems durch Gottfried von Bouillon, Königtum seines Bruders Balduin und Rückeroberung Jerusalems durch Saladin - Schlacht am Welfesholz - Verwundung und Gefangenschaft Markgraf Albrechts in Böhmen, Tod Graf Milos von Ammensleben und

25 Ibid., S. 111, S. 116.

26 Ibid., S. $118 f$.

27 Kritisch in diesem Sinne jetzt Becher, Matthias: Rex, Dux und Gens. Untersuchungen zur Entstehung des sächsischen Herzogtums im 9. und 10. Jahrhundert (Historische Studien 444), Husum 1996.

28 Folz, Robert: Le souvenir et la légende de Charlemagne dans l'Empire germanique médiéval (Publications de l'Université de Dijon 7), Paris 1950; Morrissey, Robert: L'Empereur à la Barbe Fleurie. Charlemagne dans la mythologie et l'histoire de France (Bibliothèque des Histoires), Paris 1997; Kerner, Max: Karl der Große. Entschleierung eines Mythos, Köln/Weimar/Wien 2000. 
1000 deutscher Ritter - Schlacht von Bornhöved - Einzug Kaiser Friedrichs II. in Jerusalem. Aus sächsischer Perspektive gehörten die Kaiserherrschaft Konstantins, Karls des Großen und Ottos des Großen gemeinsam mit dem Bau Magdeburgs zu den neun wichtigsten Ereignissen der Weltgeschichte. ${ }^{29}$ Die Aneignung der Größe für die eigene Welt bestimmte also im späteren Mittelalter die Erzählmotive. In ihnen durften sich die älteren Stammessagen globalisieren und imperialisieren.

\section{Schwabendiskurse}

Heinz Thomas machte in seinem viel beachteten Aufsatz "Julius Caesar und die Deutschen" die Zusammenhänge von den Entstehungsgeschichten der Deutschen und der deutschen Völker deutlich. ${ }^{30}$ Das singuläre Annolied (um 1080/85) und die breit rezipierte Kaiserchronik (um 1150) wölbten im 11. und 12. Jahrhundert über die Origines von Franken, Sachsen, Bayern und Schwaben eine neue Gründungslegende der Deutschen unter Julius Caesar. Dieser kämpfte wider diutsche land, ${ }^{31}$ erst gegen die Schwaben, dann gegen Bayern, Sachsen und Franken. Nach tapferer Gegenwehr von Caesar besiegt, retteten deutsche Helden und Krieger schließlich ihren Bezwinger. Nur mit ihrer Hilfe konnte sich Caesar in Rom durchsetzen und ehrte dafür seine Bundesgenossen, die deutschen Leute oder die Männer aus deutschen Landen. ${ }^{32}$

Im Kontext der Ursprungsgeschichten anderer Völker blieb die schwäbische Origo, mit der Herkunftsgeschichte der Bayern vielleicht überhaupt erst für den Schwaben Anno komponiert, blass. ${ }^{33}$ Übers Meer an den Fuß der Berge gelangt, hätte das wortgewandte und kriegstüchtige Volk am Berg Suêvo (Annolied) ${ }^{34}$ oder Swêrô (Kaiserchronik) ${ }^{35}$ seine Zelte aufgeschlagen und von diesem Berg den Namen Suâbo (Annolied) oder Swâbe (Kaiserchronik) ${ }^{36}$ erlangt. Eine derart knappe Anfangsgeschichte ließ sich nur wenig ausgestalten. Unabhängig davon meldete ein anonymer Autor im 12. oder in der ersten Hälfte des 13. Jahrhunderts in einer Origo der Nordschwaben (zwischen Wipper und Bode), dass dieses Volk

29 Das Buch der Welt (Anm. 5), S. 287. Zur Situierung des Textcorpus vgl. die Einleitung des Herausgebers, S. LV-LXIX.

30 Thomas, Heinz: Julius Caesar und die Deutschen. Zu Ursprung und Gehalt eines deutschen Geschichtsbewußtseins in der Zeit Gregors VII. und Heinrichs IV., in: Weinfurter, Stefan (Hg.): Die Salier und das Reich, Bd. 3: Gesellschaftlicher und ideengeschichtlicher Wandel im Reich der Salier, Sigmaringen 1991, S. 245-277.

31 Nellmann, Eberhard (Hg.): Das Annolied. Mittelhochdeutsch und Neuhochdeutsch, Stuttgart 1975 , v. 274, S. 24.

32 Ibid., v. 263-480, S. 24-38.

33 Mertens, Dieter: Spätmittelalterliches Landesbewußtsein im Gebiet des alten Schwaben, in: Werner, Matthias (Hg.): Spätmittelalterliches Landesbewußtsein in Deutschland (VuF 61), Ostfildern 2005, S. 93-156, hier S. 139.

34 Das Annolied (Anm. 31), v. 287.

35 SCHRÖDER, Edward (Hg.): Die Kaiserchronik eines Regensburger Geistlichen (MGH. Deutsche Chroniken 1,1), Hannover 1892, v. 288 f.

36 Das Annolied (Anm. 31), v. 288; Kaiserchronik (Anm. 35), v. 290. 
aus Schweden (Swein) gekommen und von dort über das Meer nach Schleswig gefahren sei. ${ }^{37}$

Schon die Kaiserchronik reicherte die knappe Herkunftssage des Annolieds mit Brennus (Prenne) an, einen frühen schwäbischen Helden, der mit Caesar über drei Stunden einen blutigen und tapferen Kampf führte. ${ }^{38}$ Ausgezeichnet sahen sich die Schwaben im Gefüge der ostfränkisch-deutschen Völker durch ihr Vorstreitrecht im Reichsheer. Lampert von Hersfeld, ein Schüler Annos von Köln, bezeugte dies erstmals 1075 für Herzog Rudolf (von Schwaben/von Rheinfelden) in der Schlacht bei Homburg an der Unstrut gegen Heinrich IV. als „besonderes Vorrecht der Schwaben" (peculiare scilicet Suevorum privilegium). ${ }^{39}$ Aus solchen Traditionssträngen erfolgte 1336 die Belehnung der Grafen von Württemberg mit der Reichssturmfahne, die im württembergischen Selbstbewusstsein ein wichtiges Herrschaftszeichen bis zum Ende des Alten Reichs blieb. ${ }^{40}$ Schon im 12. Jahrhundert erklärte die Kaiserchronik das Vorstreitrecht aus einer Verleihung durch Karl den Großen an die Schwaben, der damit die Verdienste seines Schwagers Gerold würdigte. ${ }^{41}$ So nahm Karl der Große neben Caesar die zentrale Stifterrolle im schwäbischen Geschichtsbewusstsein ein. Nur mit der Schwaben Hilfe, so verkündete es der Schwabenspiegel, habe Karl der Große Rom gewonnen und die Römer besiegt. ${ }^{42}$ Die Schwaben hätten dem Karolinger also jene Kaiserkrone gesichert, die bereits Caesar den Deutschen verdankt habe. Blickt man auf die Entstehungsdaten der hier benannten Quellen, so wird die Anreicherung des schwäbischen Selbst-

37 De origine gentis Swevorum. Qualiter Swevi terram quam nunc incolunt primum obtinuissent, in: Widukind von Corvey. Res gestae Saxonicae, ed. Hans-Eberhard Lohmann u. Paul Hirsch (MGH SS rer. Germ. in us. schol. 60), Hannover ${ }^{5} 1935$, S. 155-161, hier cap. 1 und 5, S. $155 f$.

38 Kaiserchronik (Anm. 35), v. 273-284. Die wichtigsten Forschungen zu diesen Schwabendiskursen legte vor (neben Mertens [Anm. 33]) Graf, Klaus: Aspekte zum Regionalismus in Schwaben und am Oberrhein im Spätmittelalter, in: Andermann, Kurt (Hg.): Historiographie am Oberrhein im späten Mittelalter und in der frühen Neuzeit (Oberrheinische Studien 7), Sigmaringen 1988, S. 165192; Graf, Klaus: Das ,Land' Schwaben im späten Mittelalter, in: Moraw, Peter (Hg.): Regionale Identität und soziale Gruppen im deutschen Mittelalter (Zeitschrift für historische Forschung. Beiheft 14), Berlin 1992, S. 127-164.

39 Lampert von Hersfeld, Opera, ed. Oswald Holder-Egger (MGH SS rer. Germ. in us. schol. 38), Hannover/Leipzig 1894, S. 218. Dazu Zotz, Thomas: Ethnogenese und Herzogtum in Alemannien (9.-11. Jahrhundert), in: MIÖG 108 (2000), S. 48-66, hier S. 64.

40 Erler, Adalbert: Reichsfahne, in: Handwörterbuch zur deutschen Rechtsgeschichte, Bd. 4, Berlin 1990, Sp. 567-569; BRUCKAUf, Julius: Fahnlehn und Fahnenbelehnung im alten deutschen Reiche (Leipziger Historische Abh. 3), Leipzig 1907; Thomas, Heinz: Rot und Gold - Krieg und Frieden. Die Sturmfahne des Reiches in der Bilderchronik von Kaiser Heinrichs VII. Romfahrt, in: Aufgebauer, Peter/van den Heuvel, Christine (Hg.): Herrschaftspraxis und soziale Ordnungen im Mittelalter und in der frühen Neuzeit. Ernst Schubert zum Gedenken (Veröffentlichungen der Historischen Kommission für Niedersachsen und Bremen 232), Hannover 2006, S. 17-33.

41 do verlêch der chunic Karle / Gêrolde dem helde, / daz die Swâbe von rehte / iemer suln vor vehten / durch des rîches nôt, Kaiserchronik (Anm. 35), v. 14597-14628, Zitat v. 14623-14627.

42 Eckhardt, Karl August/Eckhardt, Irmgard (Hg.): Studia iuris Suevici V: Schwabenspiegel Normalform, (Bibliotheca rerum historicarum. Studia 8), Aalen 1972, Landrecht I $₫ 32$, S. 168; Derschka, Harald Rainer: Der Schwabenspiegel, übertragen in heutiges Deutsch mit Illustrationen aus alten Handschriften, München 2002, S. $41 \mathrm{f}$. 
bewusstseins durch die Erfahrungen des staufischen König- und Kaisertums seit Konrad III. (1138-1152) und Friedrich I. Barbarossa (1152-1190) evident.

Trotz mancher Modifikationen der Schwabengeschichten vom 11. bis zum 15. Jahrhundert, deren Erforschung wir Klaus Graf und Dieter Mertens verdanken, bildete sich keine solche Komplexität heraus, wie sie in der Historiographie über die trojanische Herkunft der Franken oder die vielfältigen Wurzeln der Sachsen aus makedonischen oder skandinavischen Wurzeln begegnet. Dem ausgehenden 15. Jahrhundert entstammte dagegen eine bemerkenswerte Fiktionalisierung schwäbischer Geschichte, die in der historischen Forschung mit größter Skepsis, wenn nicht gar mit Ablehnung behandelt wurde. Dabei gehören die drei Frühdrucke der Schwäbischen Chronik des Thomas Lirer von 1485/86 aus der Ulmer Offizin des Conrad Dinckmut zu den schönsten illustrierten Historienbüchern des 15. Jahrhunderts. ${ }^{43}$ Der zweite Teil dieser Inkunabeln, eine Kaiserchronik mit schwäbischen Bezügen, schuf den lokalen wie regionalen Begebenheiten einen konventionellen historischen Rahmen, von der Sintflut über die Zerstörung Trojas, die Geschichte Roms, die mittelalterliche Kaisergeschichte bis zum Jahr 1462.

Anders löste der einem Thomas Lirer aus Rankweil zugeschriebene erste Teil den Gedächtnisentwurf einer Schwabengeschichte. ${ }^{44}$ Als historische Quelle im gängigen Sinn nahm man diese Schrift kaum in Anspruch. Dabei umgab sie die schwäbische Frühgeschichte mit ähnlichem Erfindergeschick, wie es die Trojanersagen oder Widukinds Sachsengeschichte für Franken und Sachsen taten. Während man aber den lateinischen Quellen noch ihre feinen oralen Traditionskerne gönnte, belegte man die Fabulierkunst des Schwaben mit dem Urteil „Lügenchronik“. ${ }^{4}$ Tomas Lirer schrieb seinen Text angeblich im Jahr 1133 als Knecht der Grafen von Werdenberg. Tatsächlich dürfte die Geschichte im unmittelbaren Vorfeld des Erstdrucks entstanden sein. Sie begann im Jahr 104 und erzählte vom erfundenen römischen Kaiser Kurio. Zum Christentum bekehrt, musste Kurio mit seiner Familie nach Graubünden fliehen. Hier besiegten er und seine Söhne den letzten heidnischen Herzog von Schwaben. Dessen Sohn Rumulus wurde zum ersten christlichen Herzog in Schwaben, der in seinem goldenen Wappen drei schwarze Löwen führte. ${ }^{46}$

Auf Kurios Nachkommen führte Thomas Lirer alle großen schwäbischen Adelsgeschlechter des späteren Mittelalters zurück. Seine Chronik mutierte zu einer weitgehend fiktiven Familiengeschichte der Grafen von Montfort und Werdenberg, band aber auch andere Historien ein. Unter ihnen ragten die Legende von der Kreuzauffindung durch die Kaiserin Helena in Verbindung mit dem schwäbischen Kloster Heiligenberg oder die ritterliche Reise eines Grafen von Montfort

43 Lirer, Thomas: Schwäbische Chronik, mit einem Kommentar von Peter Amelung, Leipzig 2005 [ND der Ausgabe Ulm 1468]. Zu Autor und Werk vgl. den Kommentar im Anhang.

44 Grundlegend Graf, Klaus: Exemplarische Geschichten. Thomas Lirers, Schwäbische Chronik‘ und die ,Gmündener Kaiserchronik' (Forschungen zur Geschichte der älteren deutschen Literatur 7), München 1987.

45 Beleg im Kommentar von Amelung (Anm. 43), S. 17.

46 Vgl. den Kommentar von Amelung zum unpaginierten Frühdruck, (Anm. 43), S. 18. 
an den chinesischen Kaiserhof hervor. Dort verteidigte er die Ehre der Kaiserin in einem Zweikampf und erhielt als Dank das Grabtuch Christi, das er bei der Rückreise dem Herzog von Savoyen überließ. Viel Platz beanspruchte schließlich die Liebesgeschichte zwischen Graf Albrecht von Werdenberg und der portugiesischen Königstochter Elisa. ${ }^{47}$

Solche Erzählzyklen fanden das Interesse des ausgehenden Mittelalters. In ihrer narrativen Funktion konnten sie erst wieder von einer Literaturwissenschaft gewürdigt werden, die sich von der Geschichtsquelle als Künderin historischer Wirklichkeit frei machte. In der Schwabenchronik des Thomas Lirer ging es um die schwäbische Aneignung der Welt, von der römischen Kaisergeschichte über die Heilsgeschichte bis zur adligen Existenz in der ritterlichen Adelskultur Europas. Nur im modernen Sinn changierte der Erzähler zwischen Factum und Fictum. Tatsächlich entwarf er eine einheitliche schwäbische Vergangenheit mit hohem Unterhaltungswert, ein virtuoses Spiel „mit erfundener und echter Geschichte“.48

Die Schwäbische Chronik setzte die Schwaben auf schwäbischer Erde bereits voraus. Kurio bekehrte sie lediglich zum Christentum und führte sie in die Weltgeschichte hinein. Ethnogenese oder Landnahme waren hier keine Themen mehr. Damit entdecken wir aus schwäbischer Perspektive eine entscheidende Zäsur im spätmittelalterlichen Geschichtsgedächtnis der Völker. Sie kamen nicht mehr in ihrem Land an, sondern sie waren schon da. Dieser narrative Wechsel resultierte aus der fundamentalen ethnographischen Wende in der zweiten Hälfte des Jahrhunderts, markiert durch die Entdeckung wie humanistische Rezeption der Germania des Tacitus. Dieter Mertens zeigte, wie die von Enoch d'Ascoli 1455 in die Vatikanische Bibliothek gebrachte Handschrift das Bild von der wandernden Geschichte des frühmittelalterlichen Europa veränderte. Die Rezeption begann in Italien mit Enea Silvio Piccolomini und brachte seit 1472 etwa 30 Handschriften und mehrere Drucke hervor. ${ }^{49}$

Tacitus hatte die Germanen als Ureinwohner beschrieben, nicht als Ankömmlinge: „Die Germanen selbst sind, so glaube ich jedenfalls, Ureinwohner und in keiner Weise durch Einwanderung oder gastliche Aufnahme mit fremden Völkern

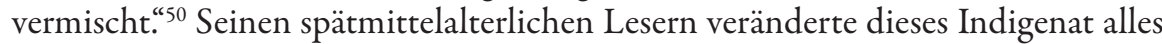
bisherige Wissen. Das Neue stand in scharfem Gegensatz zu den mittelalterlichen Wandertraditionen und zu den Ursprungsmythen der Völker aus weiten Migrationen und Eroberungen. Die Tacitus-Lektüre polarisierte nun die europäische Gelehrtenwelt. Folgenreiche Unterscheidungen in der geglaubten Vergangenheit

47 Ibid., S. 18f.

48 Ibid., S. 20. Dort auch die Hinweise auf die neuere Forschung, S. 33 f.

49 Mertens, Dieter: Die Instrumentalisierung der ,Germania des Tacitus durch die deutschen Humanisten, in: Beck/Geuenich/Steuer/Hakelberg, Gleichung (Anm. 2), S. 37-101; Krebs, Christopher B.: Negotiatio Germaniae. Tacitus' Germania und Enea Silvio Piccolomini, Giannantonio Campano, Conrad Celtis und Heinrich Bebel (Hypomnemata 158), Göttingen 2005. Vgl. auch Borst, Arno: Der Turmbau von Babel. Geschichte der Meinungen über Ursprung und Vielfalt der Sprachen und Völker, 4 Bde. in 6 Tln., Stuttgart 1957-1963, hier Bd. 3/1, S. 1033-1101.

50 TACitus, Germania, ed. Alfons STÄDELE (Tusculum Studienausgaben), Düsseldorf/Zürich 1998, cap. 2,1, S. 8 . 
germanischer oder romanischer Völker entstanden, auf die im Schlussabschnitt noch einzugehen sein wird.

Vielleicht erlangte der Augsburger Benediktiner Sigmund Meisterlin aber schon sehr bald nach der Entdeckung der Tacitus-Handschrift Kenntnis von der zentralen Botschaft der Germania? 1456/57 behauptete er in seiner lateinischen wie deutschen Augsburger Chronik, die Schwaben seien auf ihrer Erde seit unvordenklichen Zeiten verwurzelt. Trojanische Abstammung oder römische Wurzeln brachten jetzt keine Ehre mehr ein. Meisterlins Augsburg war älter als Rom. Seine Schwaben, ein Volk aus der Nachkommenschaft des Noah-Sohns Japhet, waren als erste ins Land gekommen und hatten es besiedelt. Die nach ihrer Stadt Vindelica benannten Vindeliker bildeten ein Volk der Schwaben vor der römischen Expansion. Von Schwaben gingen die Verwüstungszüge ins vorchristliche Italien aus. Hart erfuhren die Römer den furor teutonicus Lucans, und länger als alle anderen Völker widerstanden ihnen die Schwaben. Caesar musste das scharpff volck der Swaben aunüberwunden lassen. Nur durch Güte und Gaben gewann er ihre Zuneigung. Seither vollbrachten die Römer als Herren der Welt niemals große Dinge ohne Hilfe der Schwaben. Keck und treu - bereits in der römischen Kaiserzeit entstand für Meisterlin das schwäbische Selbstbewusstsein. ${ }^{51}$

Spätere bauten solche Schwabendiskurse noch weiter aus. Heinrich Bebel wurzelte sein eigenes Volk in der Heimaterde ein und schrieb einen Traktat über die Germanen als Ureinwohner. Johannes Naucler fügte seine Schwaben in die 113 generationes der Weltgeschichte ein und benutzte dafür Annius von Viterbo. So entstand das Gerüst für eine direkt auf Noah zurückreichende deutsche Vorzeit. In ihr brachte Naucler auch einen namengebenden König Suevus als Begründer der Schwaben unter. ${ }^{52}$ Die Schwabenchronik des Thomas Lirer hatte die Schwaben beim römischen Ausgriff über die Alpen bereits vorausgesetzt. Die lateinisch schreibenden Gelehrten machten sie im Übergang vom Mittelalter zur frühen Neuzeit zu ursprünglichen Bausteinen der Menschheitsgeschichte.

\section{Der ewige Deutsche}

Die Germanen als Ureinwohner! Tacitus hatte die entscheidende Fährte gelegt. Sie machte eigentlich alle ethnogenetischen Entwürfe am Ende des Mittelalters überflüssig. Als das Wissen des Römers den humanistischen Gelehrten in die Hände fiel, da entwarfen sie ein intellektuelles Laboratorium mit immer ausgreifenderen Konstrukten. Damals gingen die Geschichten der Alten verloren, die das Werden Europas noch aus fremden Migrationen und aus der engen funktionalen Verbindung Asiens und Europas erzählt hatten. Das Bewusstsein der Weite wich nun der immer gleichen deutschen Heimaterde. Mit einigem Recht hat Caspar Hirschi

51 Die Belege bei Mertens, Landesbewußtsein (Anm. 33), S. 145 f.

52 Ibid., S. 147-150; zu Heinrich Bebel vgl. Krebs, Negotiatio (Anm. 49), S. 226-250. 
darum sein viel beachtetes Buch über den Wettkampf der Nationen in ein fragendes Schlusskapitel münden lassen: „Vom Humanismus zum Holocaust?" 53

Der mittelalterliche Wechsel solch kollektiver Verankerungen - von globalen Wanderungsentwürfen des 6 . und 7. Jahrhunderts zur nationalen Homogenität von Volk und Boden im 15. und 16. Jahrhundert - hätte kaum deutlicher ausfallen können. Im 6. Jahrhundert hatte Jordanes noch permanente Wanderung und wiederholte Landnahmen als Prinzip der Volksgeschichte beschrieben: „Wie ein Bienenschwarm“ seien die Goten aus „dem Schoß der Insel“ Skandinavien nach Europa aufgebrochen. Dort, im Norden, befinde sich die „Produktionsstätte der Völker und der Geburtsort der Nationen“ (officina gentium - vagina nationum)..$^{54}$

Dieses Wissen um die Volksentstehung in Wanderschaft und Landnahme bewahrten sich auch die anderen mittelalterlichen Völker, die auf dem Boden des Imperium Romanum ihre Reiche errichteten, voran die Franken, die seit Fredegar ihre trojanischen Herkunftsmythen europäischen Völkern, Reichen, Städten und Dynastien weitergaben. ${ }^{55}$ Noch im 12. Jahrhundert erzählte der Verfasser der Historia Welforum die Lehre von der gewaltsamen Eroberung und von der trojanischen Herkunft der Welfen: „Wenn das einem unglaubwürdig erscheint, möge er die Geschichten der Völker lesen, und er wird erfahren, dass fast alle Länder gewaltsam von Fremden erobert und in Besitz genommen worden sind. Dies haben die Trojaner oft getan, nachdem sie aus ihren Gebieten vertrieben worden waren, ebenso die Goten, die Alanen, die Hunnen, die Wandalen, auch die Langobarden und die übrigen Volksstämme, am meisten aber die aus dem Norden." ${ }^{\text {"56 }}$

Als man die Geschichten nicht mehr weiter erzählte, sondern sie in den Stuben der Studierten entwarf, da wurden sie total, radikal, final. Conrad Celtis machte die Germanen zu Ureinwohnern, immer auf der gleichen Erde lebend, unter gleichem Himmel erzeugt. Über die Lage Deutschlands und die Lebensart der Deutschen schrieb er um das Jahr 1500: „Ein unbesiegtes Volk, wohlbekannt in der ganzen Welt, lebt von jeher dort, wo sich die Erde, in ihrer Kugelgestalt gekrümmt, herabneigt zum Nordpol. Geduldig erträgt es Sonnenhitze, Kälte und harte Arbeit; Müßiggang eines trägen Lebens zu erdulden leidet es nicht. Es ist ein Volk von Ureinwohnern (indigena gens), das seinen Ursprung nicht von einem anderen Geschlecht herleitet, sondern unter seinem eigenen Himmel erzeugt wurde, als Demogorgons Leib alles Erschaffene hervorgebracht hatte unter die weiten Lüfte. Germanen pflegen sie die Italiener zu nennen, die Griechen aber ,Adelphoi', weil

53 Hirschi, Wettkampf (Anm. 3), S. 489-501.

54 Jordanes, Romana et Getica, ed. Theodor Mommsen (MGH AA 5,1), Berlin 1882, S. 56, S. 60.

55 Troia. Traum und Wirklichkeit. Katalog zur Ausstellung 2001/2002, Stuttgart 2001; Kellner, Beate: Ursprung und Kontinuität. Studien zum genealogischen Wissen im Mittelalter, München 2004.

56 KöNIG, Erich (Hg.): Historia Welforum, Sigmaringen ${ }^{2} 1978$, S. 2/4. Übersetzung aus: BeCHER, Matthias (Hg.): Quellen zur Geschichte der Welfen und die Chronik Burchards von Ursberg (Ausgewählte Quellen zur deutschen Geschichte des Mittelalters. FSGA 18b), Darmstadt 2007, S. 35. 
sie gewohnt sind, wie Brüder miteinander zu leben: diesen Namen halten unsere Edlen bis heute in Ehren. ${ }^{\text {"7 }}$

Viele Traditionsstränge flossen in diesem Indigenatsdiskurs zusammen. Entscheidende Anknüpfungspunkte lieferte Tacitus mit dem erdentsprossenen Gott Tuisto und seinem Sohn Mannus, dem Ahnherrn und Begründer der Germanen (Germania, cap. 2). Die Harmonisierung des ethnographischen Wissens mit der biblischen Überlieferung verlief im Humanismus keineswegs einheitlich. ${ }^{58}$ Doch die vielen Variationen lassen sich auf wichtige Muster reduzieren. Unter dem Namen Annius von Viterbo entwarf der päpstliche Bibliothekar Giovanni Nanni (1432-1502) einen Text über den Ursprung der europäischen Völker, der angeblich vom chaldäischen Priester-Prinzen Berosus aus dem 4. oder 3. vorchristlichen Jahrhundert stammte. Dieser Pseudo-Berosus machte Tacitus' Germanengott Tuisco/Tuisto zum Adoptivsohn Noahs und zum ersten Gesetzgeber am Rhein. ${ }^{59}$ Damit hielten die Humanisten den Beleg für das im Vergleich zu den Trojanern höhere Alter der Deutschen in Händen. Im Gegensatz zu den vagen germanischen Ostgrenzen des Tacitus wurde der Pseudo-Berosus deutlicher, denn sein Tuysco war der Herrscher Sarmatiens vom Don bis zum Rhein. ${ }^{60}$ Weil dort auch die Skythen zu Noahs ersten Schülern erklärt wurden, stellten die Humanisten in der Folge die skythische Geschichte in deutsche Dienste und machten die deutsche Zivilisation zur ältesten in der Welt, geprägt von scharfer Opposition gegen alles Romanische. ${ }^{61}$ Bekämpfung und Zerstörung Roms gehörten zu den beständigen Zielen deutscher Nationalgeschichte.

Solche Strukturen wurden mannigfach variiert. Der bayerische Geschichtsschreiber Aventin baute auch noch die Gallier in deutsches Blut ein, wenn auch durch Sprach- und Blutsvermischung degeneriert. Aventin feierte auch das deutsche Drohpotential gegen alle Weltreiche. Er lobte die Mannbarkeit und Sittenreinheit der Deutschen, an der alles Fremde abpralle. Darum hielten sich deutsche Männer nicht nur von Frauen und Ausländern, sondern vor allem von Büchern

57 MÜlLER, Gernot Michael: Die ,Germania generalis` des Conrad Celtis. Studien mit Edition, Übersetzung und Kommentar (Frühe Neuzeit 67), Tübingen 2001, S. 94/95. Vgl. Krebs, Negotiatio (Anm. 49), S. 190-226.

58 Vgl. Joachimsen, Paul: Geschichtsauffassung und Geschichtsschreibung in Deutschland unter dem Einfluß des Humanismus, Bd. 1, Leipzig 1910; MUHLACK, Ulrich: Geschichtswissenschaft im Humanismus und in der Aufklärung. Die Vorgeschichte des Historismus, München 1991; Helmrath, Johannes: Die Umprägung von Geschichtsbildern in der Historiographie des europäischen Humanismus, in: LAUDAGE, Johannes (Hg.): Von Fakten und Fiktionen. Mittelalterliche Geschichtsdarstellungen und ihre kritische Aufarbeitung (Europäische Geschichtsdarstellungen 1), Köln/Weimar/ Wien 2003, S. 323-352.

59 Edition mit englischer Übersetzung bei AsHer, Ronald E.: National Myths in Renaissance France. Francus, Samothes and the Druids, Edinburgh 1993, App. I: Text and Translation of Annius Fragments Attributed to Berosus and Manetho, S. 191-233, hier S. 208. Vgl. Hirschi, Wettkampf (Anm. 3), S. 329.

60 In Europa regem Sarmatiae fecit Tuysconum a Tanai ad Rhenum, AsHer, Edition mit englischer Übersetzung (Anm. 59), S. 202.

61 Ibid., S. 198. Vgl. Hirschi, Wettkampf (Anm. 3), S. 330f. 
fern. Ihre Benutzung mache zum Narren, weil der Mann im Umgang mit Schrift und Feder verweibliche. ${ }^{62}$

Erinnerte Vergangenheit ließ die Deutschen im ausgehenden Mittelalter also zu harten und ewigen Männern werden. Die Kreation einer völkischen Dauerhaftigkeit erfolgte ausgerechnet in einer Zeit, in der das Heilige Römische Reich deutscher Nation mit seinen universalen Ansprüchen in der europäischen Mächtegesellschaft auf das Normalmaß eines Teils nivelliert worden war. Wie auch immer die Kaiser mit der Beschränkung ihrer globalen Ansprüche umgehen mochten die gelehrten Humanisten schufen sich ihre eigene Vorstellungswelt vom Vorrang des deutschen Volkes, das seit unvordenklichen Zeiten auf seiner eigenen Scholle wohnte. Wanderung, Landnahme oder Ethnogenese fielen aus diesem deutschen Selbstbewusstsein heraus. Die Folgen für die Weiterentwicklung nationaler Stereotype in der Neuzeit waren beträchtlich. Es machte nämlich durchaus einen Unterschied, ob ein Volk seine Geschichte aus fremden Ursprüngen oder aus der ewig gleichen Heimaterde, also der Verknüpfung von Blut und Boden, entwickelte.

\section{Schluss}

Warum, so ließe sich fragen, wurden im Kontext dieses Bandes solch pittoreske, ja monströse Bilder des Spätmittelalters ausgebreitet? Keine der behandelten Quellen bereichert die aktuellen Forschungen um Völker, Reiche und Namen des früheren Mittelalters. Will man - über ein buntes Allerlei hinaus - der modernen ethnogenetischen Forschung überhaupt Diskussionsstoff anbieten, so ließen sich drei Punkte herausstellen:

1.) Ethnogenetische Erinnerung blieb immer fiktional, im 7., im 10. wie im 15. Jahrhundert. Vielleicht ist ihre Erforschung bis zu einem gewissen Grad aussichtslos, weil sie keine ,wahren Gedächtniskerne' von Migration und politischer Verbandsbildung transportierte, sondern immer nur Vergangenheitskonstrukte aus späteren Sehnsüchten heraus entwickelt. So könnte es für die Suche nach den Ursprüngen der Sachsen, der Schwaben oder der Deutschen fast gleichgültig erscheinen, ob man Widukind von Corvey oder die Magdeburger Schöppenchronik, ob man Sigmund Meisterlin oder Thomas Lirer, ob man das Annolied oder Aventin liest. In jedem Fall stößt man nur auf spätere Verformungen einer geglaubten Vergangenheit.

2.) Die Globalisierung des Wissens mit einer neuen Informationsflut und elaborierten Kommunikationsweisen brachte im 15. und 16. Jahrhundert keine vertieften Erkenntnisse zur frühmittelalterlichen Ethnogenese, sondern glänzte allenfalls durch den Mut zu simplifizierenden Deutungen. Der kühne Zugriff auf die ganze Weltgeschichte zeitigte damals keinen qualitativen Fortschritt.

62 Die Belege ibid., S. 331-338. 
Er beförderte dagegen die kreative Kraft zur intellektuellen Schaumschlägerei jenseits vernünftiger Evidenz.

3.) Die ethnogenetische Forschung räumte seit dem Zweiten Weltkrieg zwar manche völkischen Schlacken beiseite und erschütterte die überzeitlichen Stilisierungen der deutschen Völker wie der Deutschen. Dabei berücksichtigte sie vielleicht die permanente Kraft sich beständig verformender Modelle seit dem 14. Jahrhundert zu wenig. Jetzt wird sie plötzlich vom kaum verhüllten Faschismusvorwurf gegen ethnogenetische Geschichtsforschung aufgeschreckt. Wissenschaftsgeschichte darf nicht bei den nationalen oder nationalistischen Deutungskartellen des 19. und 20. Jahrhunderts beginnen. Sie muss die langen Verformungen der Erinnerung über alle Zeiten bedenken und wird dabei die prägende Kraft des ausgehenden Mittelalters auf die Erkenntnisfähigkeit und die Gegensätze in den europäischen Kulturen entdecken. 
